\title{
Neurogenic chronic idiopathic intestinal pseudo-obstruction, patent ductus arteriosus, and thrombocytopenia segregating as an $\mathrm{X}$ linked recessive disorder
}

\author{
D R FitzPatrick, L Strain, A E Thomas, D G D Barr, A Todd, N M Smith, W G Scobie
}

Department of Clinical Genetics, Molecular

Medicine Centre, Western General Hospital, Edinburgh EH4 2XU, UK

D R FitzPatrick

Department of Human Genetics, Molecular Medicine Centre, Western General Hospital, Edinburgh EH4 2XU, UK D R FitzPatrick L Strain

Department of Paediatric

Haematology, Royal

Hospital for Sick Children, Edinburgh EH9 1LF, UK

A E Thomas

Department of Medicine, Royal Hospital for Sick Children, Edinburgh EH9 1LF, UK

D G D Barr

Scottish Blood

Transfusion Service, Royal Infirmary,

Edinburgh, UK

A Todd

Department of Pathology, Royal Hospital for Sick Children, Edinburgh EH9 1LF, UK N M Smith

Department of Surgery, Royal Hospital for Sick Children, Edinburgh EH9 1LF, UK

W G Scobie

Correspondence to: Dr FitzPatrick.

Received 25 October 1996 Revised version accepted for publication 7 February 1997

\begin{abstract}
We present a family with three affected males in two generations with congenital neurogenic chronic idiopathic intestinal pseudo-obstruction (CIIP), patent ductus arteriosus, and large platelet thrombocytopenia apparently segregating as an $X$ linked recessive disorder. The pattern of segregation of DNA markers within the family is consistent with linkage to the previously described neurogenic CIIP (CIIPX) locus at Xq28. This combination may represent a new contiguous gene disorder and appears to have a good prognosis with supportive therapy.

(FMed Genet 1997;34:666-669)
\end{abstract}

Keywords: X chromosome; pseudo-obstruction; patent ductus arteriosus; thrombocytopenia

Chronic idiopathic intestinal pseudoobstruction (CIIP) may present at any age and is diagnosed by finding radiological, surgical, or manometric evidence of abnormal bowel motility causing clinical features of intestinal obstruction with no mechanical aetiology. ${ }^{12}$ Congenital CIIP is a rare, often fatal, ${ }^{3}$ cause of abdominal distension in infancy and can be categorised, on the basis of histological examination of a full thickness intestinal biopsy, into three groups, neurogenic $(28 \%)$, myopathic $(36 \%)$, and unclassifiable $(36 \%)$. Recently, an $\mathrm{X}$ linked recessive neurogenic form of congenital CIIP (CIIPX) associated with pyloric hypertrophy and gut malrotation has been shown to cosegregate with DNA markers at Xq28 in a large Italian family. CIIPX is not associated with any other non-gastrointestinal dysmorphic features. Here we present a family with an apparently new $X$ linked disorder characterised by congenital neurogenic CIIP, patent ductus arteriosus (PDA), facial dysmorphism, and an unusual large platelet thrombocytopenia. We also discuss similarities with three previously reported male infants ${ }^{67}$ and possible disease mechanisms in this family.

\section{Case reports}

CASE 1

The proband is III.1 (fig 1), the first child of healthy, non-consanguineous Scottish parents. $\mathrm{He}$ was born at term weighing $3970 \mathrm{~g}$ after an uneventful pregnancy. At 4 days he was assessed for bilious vomiting and abdominal distension and a laparotomy performed at 10 days showed malrotation with a narrow pedicle, which was corrected. $\mathrm{He}$ also required operative ligation of a patent ductus arteriosus (PDA) at the age of 24 days. A post-laparotomy barium meal showed delayed passage with no obstruction. At 3 months he presented with chronic diarrhoea and vomiting and an intestinal biopsy at that time showed neuronal intestinal dysplasia (fig 2) suggestive of a diagnosis of neurogenic CIIP. His feeding problems were profound throughout the first year of life and he initially required total parenteral nutrition with subsequent nasogastric (NG) tube feeding. Chronic thrombocytopenia (32-104 $\times$ $\left.10^{9} / 1\right)$ with no bleeding problem had also been noted since birth. A bone marrow aspiration at 6 months of age was normal with plentiful megakaryocytes. He had further operations for an undescended right testis and infantile esotropia. When last reviewed at 6 years of age

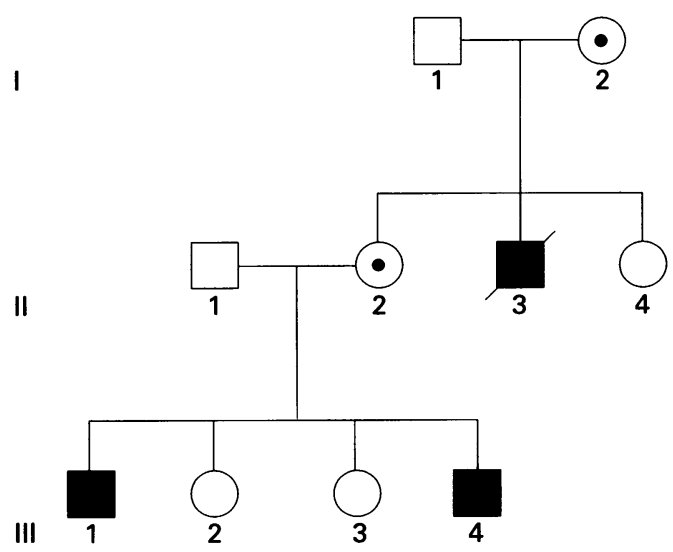

Figure 1 Family pedigree showing affected subjects II.3, III.1, and III. 4 related through a phenotypically normal female, II.2. I. 3 died in the neonatal period, but the cause of death is not known. 


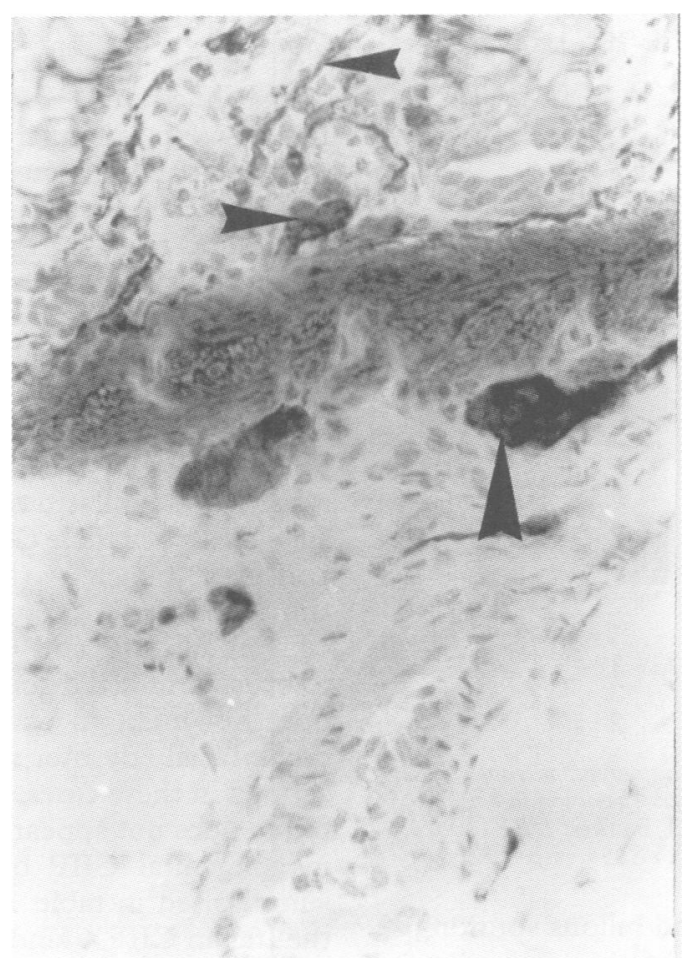

Figure 2 Photomicrograph showing ileal mucosa with aberrant nerve fibres and ganglion cells in the lamina propria (small arrowheads). Large groups of ganglion cells in normal submucosal location beneath muscularis mucosae are also present (large arrowhead). Acetylcholinesterase stain.

(fig 3A) he was generally in good health with near normal bowel function and doing well at a normal school. He had mild craniofacial dysmorphism with a rather smooth philtrum, a large jaw, and low set ears. Chromosome analysis was normal.

CASE 2

Case 2 (III.4) is the younger brother of case 1 and the fourth child of their parents. Case 2 also has two older sisters (III.2, III.3) who are both completely well. He was born at 38 weeks' gestation weighing $3827 \mathrm{~g}$ after a normal pregnancy. He had early neonatal jaundice and was noted to have feeding difficulties which necessitated NG tube feeding. He was also noted to be facially dysmorphic (fig 3B) with downward slanting palpebral fissures, hypertelorism, and low set ears. At 2 weeks of age he had an episode of abdominal distension and visible peristalsis. Barium studies showed slow passage of the contrast media through the small bowel with no evidence of mechanical obstruction. A rectal biopsy at that time was too superficial to diagnose neuronal intestinal dysplasia; however, the clinical features were consistent with the diagnosis of CIIP. He was also noted to have left sided hydronephrosis and a PDA which was ligated at 22 days. He has been persistently mildly thrombocytopenic $(60-140 \times$ $10^{9} / 1$ ) with large platelets noted on inspection of the blood film (fig 4) but no bleeding problems. His clinical picture over the first 9 months of life has been very similar to his older brother with poor feeding (he continues to be fed via nasogastric tube) and infrequent episodes of abdominal distension and vomiting. His neurodevelopmental profile is normal.
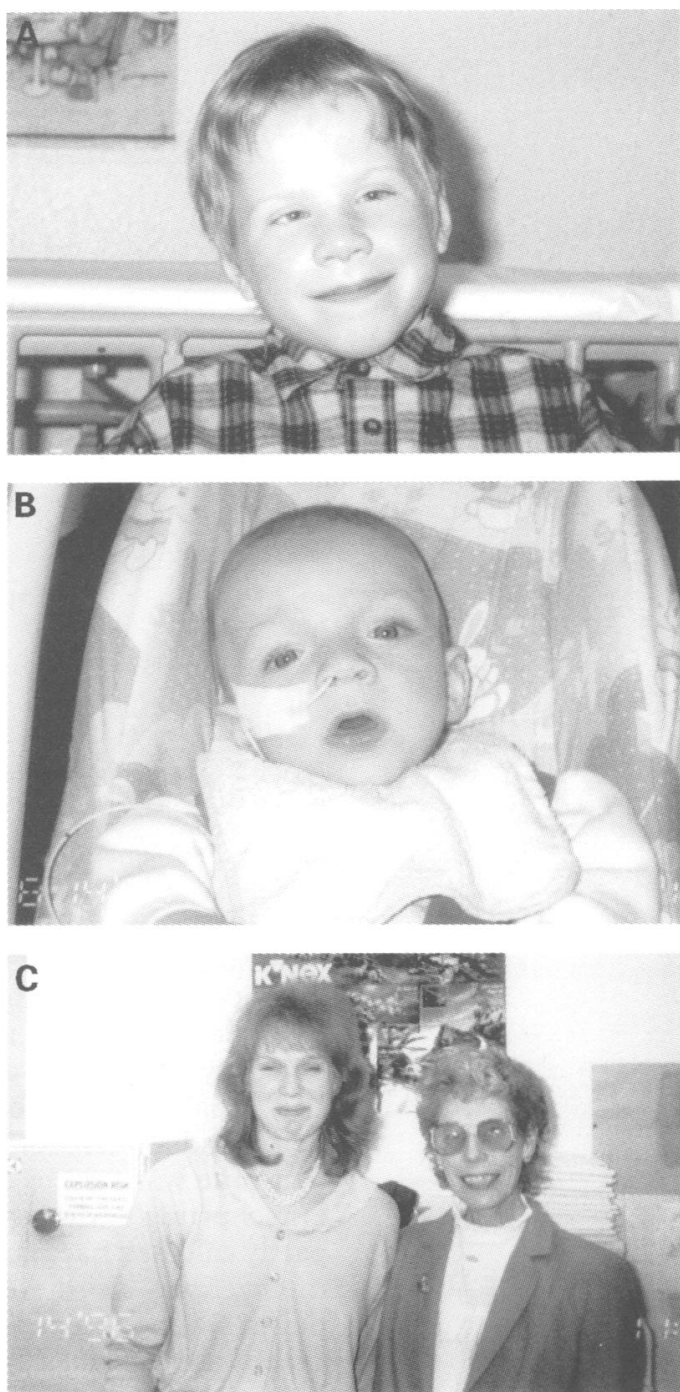

Figure 3 Facial photographs of affected subjects and obligate carrier women. (A) Facial photograph of case 1 at the age of 6 years. (B) Case 2 at the age of 8 months with downward slanting palpebral fissures and hypertelorism; a NG tube is required for feeding problems. (C) Mother (left) and maternal grandmother (right) of cases 1 and 2.

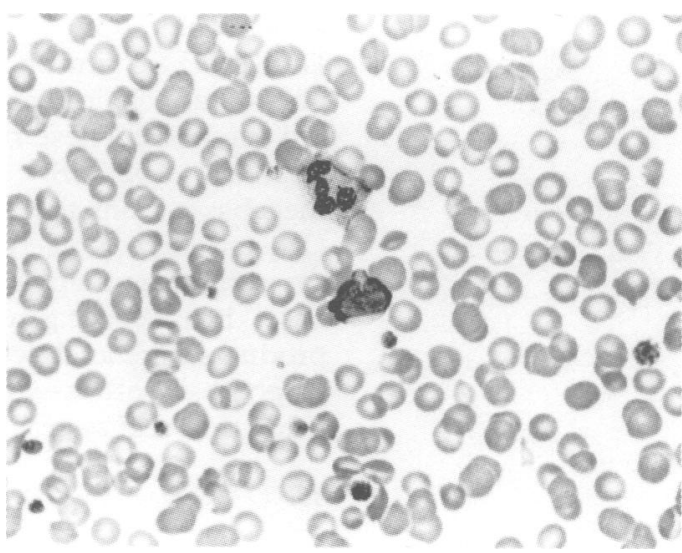

Figure 4 A peripheral blood film from case 2 shows large but not giant platelets with normal granulation in association with a mild thrombocytopenia. This is in contrast to Wiskott-Aldich syndrome where the platelets are small. A normal neutrophil and lymphocyte are also shown.

\section{CASE 3}

Case 3 (II.3) is the maternal uncle of cases 1 and 2 and was born in 1961 as the first child of healthy, non-consanguineous Scottish parents. $\mathrm{He}$ was born at term weighing $3800 \mathrm{~g}$. He was admitted to hospital at the age of 3 days with a 


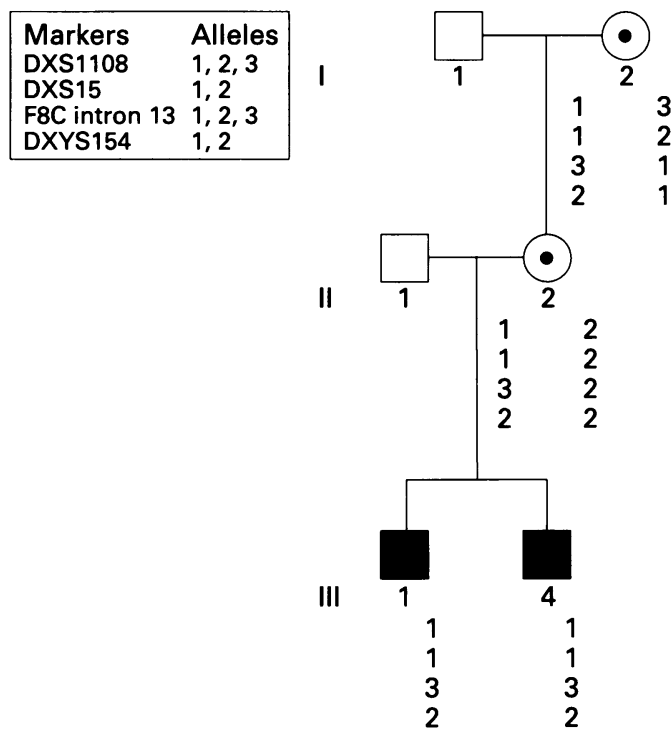

Figure 5 Segregation of Xq28 haplotypes in subjects I.2, II.2, III.1, and III. 4. The numbers (from top to bottom) are designated alleles at the $(C A)_{n}$ markers $D X S 1108$, DXS15, F8C intron 13, and DXYS154, respectively.

history of poor feeding and bilious vomiting. A laparotomy at the age of 16 days showed malrotated, short midgut with dilatation of the small bowel. He died at the age of 5 weeks and necropsy showed a patent ductus arteriosus and atrial septal defect with distended small bowel and a microcolon. Intestinal histology at that time was said to "exclude the presence of Hirschprung's disease". A $3 \mathrm{~cm}$ diameter partly calcified haemangioma of the left lobe of the liver was also noted. Craniofacial dysmorphism was not mentioned and no facial photographs are available.

\section{DNA studies}

DNA MARKERS

DNA samples were available for analysis on subjects I.2, II.2, III.1, and III.4. Fluorescently labelled primers were used to amplify highly polymorphic $(C A)_{n}$ repeat markers DXS1 108, DXS15, F8C intron 13, and DXYS154 by polymerase chain reaction (PCR) using standard methods. These markers map to Xq28 at $162.261,162.458,163.596$, and 163.669 megabases from the Xp telomere respectively

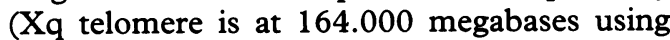
the composite map at Genetic Location Database, http://cedar.genetics.soton.ac.uk). PCR products were analysed by acrylamide gel electrophoresis with laser detection of fluorescence using the ALF system (Pharmacia).

Table 1 Comparison of clinical features of affected subjects
RESULTS

These markers were chosen because they have been linked to CIIPX in one large Italian family. ${ }^{5}$ No submicroscopic deletions were detected in the affected boys using these markers. However, a grandmaternal Xq28 haplotype was found to cosegregate with the disease (fig 5) giving a maximum lod score of 0.602 at $\theta=0.0$.

\section{Discussion}

The affected subjects in this family have an unusual pattern of clinical features which show remarkable similarities to a previously reported single case of a male child with congenital CIIP and giant platelets ${ }^{6}$ (table 1). There are also similarities to a report of two brothers with congenital CIIP and PDA which was thought to be an $\mathrm{X}$ linked disorder, although this report does not mention platelet abnormalities. ${ }^{7}$ No craniofacial dysmorphic features were described in these cases. Interestingly, gut malrotation does not appear to be a common feature in congenital CIIP but is seen in $5 / 6$ cases summarised in table 1 and several subjects in the Italian CIIPX kindred. ${ }^{5}$ This malformation could, therefore, prove to be a useful phenotypic marker for an $\mathrm{X}$ linked form of the disease.

In the present family the affected subjects are male and are related through a normal female (fig 3C) suggesting $X$ linked recessive inheritance. In this respect it is interesting that I. 2 had an older brother who died in the late neonatal period, although no clinical details are available. Sex modified expression of an autosomal dominant trait or a cryptic chromosomal translocation could mimic this inheritance pattern. An X linked form of CIIP (CIIPX) associated with bowel malrotation and pyloric stenosis has recently been mapped to Xq28 with a maximum lod score of 2.32 at $\theta=0.0$ with DXS154. The DNA marker results in our family are consistent with linkage to this marker although the lod score generated from such a small family is obviously non-significant ( $\sim: 1$ odds of linkage at the single locus tested).

The apparently disparate clinical features seen in the affected subjects in this report may be the result of a microdeletion in Xq28 which has inactivated CIIPX and neighbouring genes involved in platelet production and ductus arteriosus closure. Against this thought is the fact that there have been no families described with an X linked form of PDA, although PDA does occur as part of the spectrum of severe

\begin{tabular}{|c|c|c|c|c|c|c|}
\hline \multirow[b]{2}{*}{ Features } & \multirow[b]{2}{*}{ Case 1} & \multirow[b]{2}{*}{ Case 2} & \multirow[b]{2}{*}{ Case 3} & \multicolumn{2}{|c|}{ Harris et al ${ }^{7}$} & \multirow[b]{2}{*}{ Pollock et al } \\
\hline & & & & Case 1 & Case 2 & \\
\hline Sex & $\mathbf{M}$ & $\mathbf{M}$ & $\mathbf{M}$ & $\mathbf{M}$ & $\mathbf{M}$ & $\mathbf{M}$ \\
\hline CIIP & + & + & + & + & + & + \\
\hline Malrotation & + & - & + & + & + & + \\
\hline Pyloric stenosis & - & - & - & - & - & + \\
\hline PDA & + & + & + & + & + & + \\
\hline Thrombocytopenia & + & + & $?$ & $?$ & $?$ & + \\
\hline Hydronephrosis & - & + & - & - & - & $?$ \\
\hline Esotropia & + & - & $?$ & $?$ & $?$ & $?$ \\
\hline Undescended testes & + & - & $?$ & - & - & $?$ \\
\hline Normal intelligence & + & + & $?$ & $?$ & $?$ & $?$ \\
\hline
\end{tabular}


malformations in the thoracoabdominal syndrome (MIM 313850). Three different types of $\mathrm{X}$ linked thrombocytopenia have been described: X linked thrombocytopenia (MIM 313900 , maps to the same region of $\mathrm{Xp}$ as Wiskott-Aldrich syndrome), thrombocytopenia with raised serum IgA and renal disease (MIM 314000, single family), and thrombocytopenia, platelet dysfunction, haemolysis, and imbalanced globin synthesis (MIM 314050, single family). None of these is associated with large platelets, indeed MIM 313900 causes reduced platelet volume, and all cause significant bleeding diatheses.

Another possible explanation for the apparently unrelated clinical features in these children is that the CIIPX gene may have a role in the developmental regulation of multiple systems with different mutations causing a different spectrum of abnormalities. The influence of prostaglandin $\mathrm{E}$ on platelet function and ductus closure is well known and it is possible that mutations in the prostaglandin biosynthetic or regulatory genes may result in a phenotype similar to this family. However, no genes in this pathway have been identified on the $\mathrm{X}$ chromosome.

We would like to acknowledge the involvement and support of the family in this work, which was funded by the Western General Hospital NHS Trust and the Edinburgh Sick Children's NHS Trust. We also thank Dr Susan Holloway for help with lod score calculation.

1 Miglioli M, Pironi L. Chronic intestinal pseudoobstruction Clin Nutrit 1995;14:21-3.

2 Ghosh S, Eastwood MA Primary chronic intestinal pseudoobstruction - an update. Postgrad Med $\mathcal{F}$ 1994;70:65pseud.

3 Huang YC, Lee HG, Huang FY, et al. Neonatal-onset chronic intestinal pseudoobstruction syndrome. Clin Pediatr 1995;34:241-7.

4 Fell JME, Smith VV, Milla PJ. Infantile chronic idiopathic intestinal pseudoobstruction - the role of small-intestinal manometry as a diagnostic-tool and prognostic indicator. Gut 1996;39:306-11.

5 Auricchio A, Brancolini V, Casari G, et al. The locus for a novel syndromal form of neuronal intestinal pseudoobstruction maps to Xq28. Am f Hum Genet 1996;58:743-8.

6 Pollock I, Holmes SJK, Patton MA, Hamilton PA, Stacey TE. Congenital intestinal pseudo-obstruction associated with a giant platelet disorder. $\mathcal{F}$ Med Genet 1991;28:495-6.

7 Harris DJ, Ashkraft KW, Beatty EC, Holder TM, Leonidas JC. Natal teeth, patent ductus arteriosus and intestinal pseudo-obstruction: a lethal syndrome in the newborn. pseudo-obstruction: a lethal 